$1-1-2006$

\title{
Electron scattering from high-momentum neutrons in deuterium
}

\author{
A. V. Klimenko
}

Angela Biselli

Fairfield University, abiselli@fairfield.edu

CLAS Collaboration

Follow this and additional works at: https://digitalcommons.fairfield.edu/physics-facultypubs

Copyright American Physical Society Publisher final version available at http://prc.aps.org/pdf/ PRC/v73/i3/e035212

\section{Peer Reviewed}

\section{Repository Citation}

Klimenko, A. V.; Biselli, Angela; and CLAS Collaboration, "Electron scattering from high-momentum neutrons in deuterium" (2006). Physics Faculty Publications. 80.

https://digitalcommons.fairfield.edu/physics-facultypubs/80

\section{Published Citation}

A. V. Klimenko et al. [CLAS Collaboration], "Electron scattering from high-momentum neutrons in deuterium", Phys. Rev. C 73, 035212 (2006) DOI: 10.1103/PhysRevC.73.035212

This item has been accepted for inclusion in DigitalCommons@Fairfield by an authorized administrator of DigitalCommons@Fairfield. It is brought to you by DigitalCommons@Fairfield with permission from the rightsholder(s) and is protected by copyright and/or related rights. You are free to use this item in any way that is permitted by the copyright and related rights legislation that applies to your use. For other uses, you need to obtain permission from the rights-holder(s) directly, unless additional rights are indicated by a Creative Commons license in the record and/or on the work itself. For more information, please contact digitalcommons@fairfield.edu. 


\section{Electron scattering from high-momentum neutrons in deuterium}

A. V. Klimenko, ${ }^{27, *}$ S. E. Kuhn, ${ }^{27, \dagger}$ C. Butuceanu,${ }^{38}$ K. S. Egiyan, ${ }^{39}$ K. A. Griffioen, ${ }^{38}$ G. Adams,${ }^{29}$ P. Ambrozewicz, ${ }^{9}$ M. Anghinolfi, ${ }^{15}$ G. Asryan, ${ }^{39}$ H. Avakian, ${ }^{34}$ H. Bagdasaryan, ${ }^{27,39}$ N. Baillie, ${ }^{38}$ J. P. Ball, ${ }^{1}$ N. A. Baltzell, ${ }^{33}$ S. Barrow, ${ }^{10}$ V. Batourine,${ }^{20}$ M. Battaglieri, ${ }^{15}$ I. Bedlinskiy, ${ }^{18}$ M. Bektasoglu, ${ }^{31}$ M. Bellis, ${ }^{3,29}$ N. Benmouna, ${ }^{11}$ A. S. Biselli, ${ }^{3,29}$ S. Bouchigny, ${ }^{16}$ S. Boiarinov, ${ }^{34}$ R. Bradford,${ }^{3}$ D. Branford, ${ }^{8}$ W. K. Brooks, ${ }^{34}$ S. Bültmann, ${ }^{27}$ V. D. Burkert ${ }^{34}$ J. R. Calarco, ${ }^{24}$ S. L. Careccia, ${ }^{27}$ D. S. Carman, ${ }^{26}$ A. Cazes,${ }^{33}$ S. Chen,${ }^{10}$ P. L. Cole,${ }^{13,34}$ P. Coltharp,${ }^{10}$ D. Cords,${ }^{34, \ddagger}$ P. Corvisiero,,${ }^{15}$ D. Crabb,${ }^{37}$ J. P. Cummings, ${ }^{29}$ N. B. Dashyan, ${ }^{39}$ R. DeVita,${ }^{15}$ E. De Sanctis, ${ }^{14}$ P. V. Degtyarenko,${ }^{34}$ H. Denizli, ${ }^{28}$ L. Dennis, ${ }^{10}$

K. V. Dharmawardane, ${ }^{27}$ C. Djalali, ${ }^{33}$ G. E. Dodge, ${ }^{27}$ J. Donnelly, ${ }^{12}$ D. Doughty, ${ }^{6,34}$ M. Dugger, ${ }^{1}$ S. Dytman, ${ }^{28}$ O. P. Dzyubak ${ }^{33}$ H. Egiyan,${ }^{34,38,8}$ L. Elouadrhiri, ${ }^{34}$ P. Eugenio, ${ }^{10}$ R. Fatemi, ${ }^{37}$ G. Fedotov, ${ }^{23}$ R. G. Fersch, ${ }^{38}$ R. J. Feuerbach, ${ }^{34}$ H. Funsten, ${ }^{38}$ M. Garçon, ${ }^{5}$ G. Gavalian, ${ }^{24,27}$ G. P. Gilfoyle, ${ }^{32}$ K. L. Giovanetti, ${ }^{19}$ F. X. Girod, ${ }^{5}$ J. T. Goetz, ${ }^{2}$ A. Gonenc, ${ }^{9}$ C. I. O. Gordon, ${ }^{12}$ R. W. Gothe, ${ }^{33}$ M. Guidal, ${ }^{16}$ M. Guillo, ${ }^{33}$ N. Guler, ${ }^{27}$ L. Guo,${ }^{34}$ V. Gyurjyan, ${ }^{34}$ C. Hadjidakis, ${ }^{16}$

R. S. Hakobyan, ${ }^{4}$ J. Hardie,${ }^{6,34}$ F. W. Hersman, ${ }^{24}$ K. Hicks, ${ }^{26}$ I. Hleiqawi,${ }^{26}$ M. Holtrop,${ }^{24}$ C. E. Hyde-Wright,${ }^{27}$ Y. Ilieva, ${ }^{11}$ D. G. Ireland, ${ }^{12}$ B. S. Ishkhanov ${ }^{23}$ M. M. Ito, ${ }^{34}$ D. Jenkins, ${ }^{36}$ H. S. Jo, ${ }^{16}$ K. Joo,${ }^{7,34}$ H. G. Juengst, ${ }^{11, \|}$ J. D. Kellie, ${ }^{12}$ M. Khandaker, ${ }^{25}$ W. Kim, ${ }^{20}$ A. Klein, ${ }^{27}$ F. J. Klein, ${ }^{4}$ M. Kossov, ${ }^{18}$ L. H. Kramer, ${ }^{9,34}$ V. Kubarovsky, ${ }^{29}$ J. Kuhn, ${ }^{3,29}$ S. V. Kuleshov, ${ }^{18}$ J. Lachniet, ${ }^{3}$ J. M. Laget, ${ }^{5,34}$ J. Langheinrich, ${ }^{33}$ D. Lawrence, ${ }^{22} \mathrm{Ji} \mathrm{Li}^{29}{ }^{29}$ K. Livingston, ${ }^{12}$ S. McAleer, ${ }^{10}$ B. McKinnon, ${ }^{12}$ J. W. C. McNabb, ${ }^{3}$ B. A. Mecking, ${ }^{34}$ S. Mehrabyan, ${ }^{28}$ J. J. Melone, ${ }^{12}$ M. D. Mestayer, ${ }^{34}$ C. A. Meyer, ${ }^{3}$ T. Mibe,${ }^{26}$ K. Mikhailov, ${ }^{18}$ R. Minehart ${ }^{37}$ M. Mirazita, ${ }^{14}$ R. Miskimen, ${ }^{22}$ V. Mokeev ${ }^{23}$ L. Morand, ${ }^{5}$ S. A. Morrow, ${ }^{5,16}$ J. Mueller, ${ }^{28}$ G. S. Mutchler, ${ }^{30}$ P. Nadel-Turonski, ${ }^{11}$ J. Napolitano, ${ }^{29}$ R. Nasseripour,,${ }^{93}$ S. Niccolai, ${ }^{11,16}$ G. Niculescu, ${ }^{19,26}$ I. Niculescu, ${ }^{19,34}$ B. B. Niczyporuk, ${ }^{34}$ R. A. Niyazov, ${ }^{27,34}$ M. Nozar,${ }^{34}$ G. V. O'Rielly, ${ }^{11}$ M. Osipenko,,${ }^{15,23}$ A. I. Ostrovidov, ${ }^{10}$ K. Park ${ }^{20}$ E. Pasyuk, ${ }^{1}$ C. Paterson, ${ }^{12}$ J. Pierce, ${ }^{37}$ N. Pivnyuk, ${ }^{18}$ D. Pocanic,${ }^{37}$ O. Pogorelko, ${ }^{18}$ S. Pozdniakov, ${ }^{18}$

B. M. Preedom, ${ }^{33}$ J. W. Price, ${ }^{2}$ Y. Prok,${ }^{37,9}$ D. Protopopescu, ${ }^{12,24}$ B. A. Raue,,${ }^{94}$ G. Riccardi, ${ }^{10}$ G. Ricco, ${ }^{15}$ M. Ripani, ${ }^{15}$ B. G. Ritchie, ${ }^{1}$ F. Ronchetti, ${ }^{14}$ G. Rosner, ${ }^{12}$ P. Rossi,${ }^{14}$ F. Sabatié,${ }^{5}$ C. Salgado, ${ }^{25}$ J. P. Santoro, ${ }^{4}$ V. Sapunenko, ${ }^{34}$ R. A. Schumacher, ${ }^{3}$ V. S. Serov ${ }^{18}$ Y. G. Sharabian, ${ }^{34}$ A. V. Skabelin, ${ }^{21}$ E. S. Smith, ${ }^{34}$ L. C. Smith,${ }^{37}$ D. I. Sober, ${ }^{4}$ A. Stavinsky, ${ }^{18}$ S. S. Stepanyan, ${ }^{20}$ S. Stepanyan, ${ }^{34}$ B. E. Stokes,${ }^{10}$ P. Stoler ${ }^{29}$ S. Strauch, ${ }^{11}$ M. Taiuti, ${ }^{15}$ D. J. Tedeschi, ${ }^{33}$ U. Thoma,,${ }^{17,34, * *}$ A. Tkabladze, ${ }^{26}$ S. Tkachenko, ${ }^{27}$ L. Todor, ${ }^{3}$ C. Tur, ${ }^{33}$ M. Ungaro, ${ }^{7,29}$ M. F. Vineyard ${ }^{32,35}$ A. V. Vlassov, ${ }^{18}$ L. B. Weinstein, ${ }^{27}$ D. P. Weygand, ${ }^{34}$ M. Williams, ${ }^{3}$ E. Wolin,${ }^{34}$ M. H. Wood,${ }^{33, \dagger \dagger}$ A. Yegneswaran, ${ }^{34}$ L. Zana, ${ }^{24}$ J. Zhang, ${ }^{27}$ and B. Zhao ${ }^{7}$ (CLAS Collaboration)

${ }^{1}$ Arizona State University, Tempe, Arizona 85287-1504, USA

${ }^{2}$ University of California at Los Angeles, Los Angeles, California 90095-1547, USA

${ }^{3}$ Carnegie Mellon University, Pittsburgh, Pennsylvania 15213, USA

${ }^{4}$ Catholic University of America, Washington, DC 20064, USA

${ }^{5}$ CEA-Saclay, Service de Physique Nucléaire, F-91191 Gif-sur-Yvette, Cedex, France

${ }^{6}$ Christopher Newport University, Newport News, Virginia 23606, USA

${ }^{7}$ University of Connecticut, Storrs, Connecticut 06269, USA

${ }^{8}$ Edinburgh University, Edinburgh EH9 3JZ, United Kingdom

${ }^{9}$ Florida International University, Miami, Florida 33199, USA

${ }^{10}$ Florida State University, Tallahassee, Florida 32306, USA

${ }^{11}$ George Washington University, Washington, DC 20052, USA

${ }^{12}$ University of Glasgow, Glasgow G12 8QQ, United Kingdom

${ }^{13}$ Idaho State University, Pocatello, Idaho 83209, USA

${ }^{14}$ INFN, Laboratori Nazionali di Frascati, Frascati, Italy ${ }^{15}$ INFN, Sezione di Genova, I-16146 Genova, Italy

${ }^{16}$ Institut de Physique Nucleaire Orsay, Orsay, France

${ }^{17}$ Institute für Strahlen und Kernphysik, Universität Bonn, Germany

${ }^{18}$ Institute of Theoretical and Experimental Physics, RU-117259 Moscow, Russia

${ }^{19}$ James Madison University, Harrisonburg, Virginia 22807, USA

${ }^{20}$ Kyungpook National University, Daegu 702-701, South Korea

${ }^{21}$ Massachusetts Institute of Technology, Cambridge, Massachusetts 02139, USA

${ }^{22}$ University of Massachusetts, Amherst, Massachusetts 01003, USA

${ }^{23}$ Moscow State University, General Nuclear Physics Institute, RU-119899 Moscow, Russia

${ }^{24}$ University of New Hampshire, Durham, New Hampshire 03824, USA

${ }^{25}$ Norfolk State University, Norfolk, Virginia 23504, USA

${ }^{26}$ Ohio University, Athens, Ohio 45701, USA

${ }^{27}$ Old Dominion University, Norfolk, Virginia 23529, USA

${ }^{28}$ University of Pittsburgh, Pittsburgh, Pennsylvania 15260, USA

${ }^{29}$ Rensselaer Polytechnic Institute, Troy, New York 12180, USA

${ }^{30}$ Rice University, Houston, Texas 77005, USA 


\author{
${ }^{31}$ Sakarya University, Sakarya, Turkey \\ ${ }^{32}$ University of Richmond, Richmond, Virginia 23173, USA \\ ${ }^{33}$ University of South Carolina, Columbia, South Carolina 29208, USA \\ ${ }^{34}$ Thomas Jefferson National Accelerator Facility, Newport News, Virginia 23606, USA \\ ${ }^{35}$ Union College, Schenectady, New York 12308, USA \\ ${ }^{36}$ Virginia Polytechnic Institute and State University, Blacksburg, Virginia 24061, USA \\ ${ }^{37}$ University of Virginia, Charlottesville, Virginia 22901, USA \\ ${ }^{38}$ College of William and Mary, Williamsburg, Virginia 23187, USA \\ ${ }^{39}$ Yerevan Physics Institute, 375036 Yerevan, Armenia \\ (Received 14 October 2005; published 24 March 2006)
}

\begin{abstract}
We report results from an experiment measuring the semiinclusive reaction ${ }^{2} \mathrm{H}\left(e, e^{\prime} p_{s}\right)$ in which the proton $p_{s}$ is moving at a large angle relative to the momentum transfer. If we assume that the proton was a spectator to the reaction taking place on the neutron in deuterium, the initial state of that neutron can be inferred. This method, known as spectator tagging, can be used to study electron scattering from high-momentum (off-shell) neutrons in deuterium. The data were taken with a $5.765 \mathrm{GeV}$ electron beam on a deuterium target in Jefferson Laboratory's Hall B, using the CEBAF large acceptance spectrometer. A reduced cross section was extracted for different values of final state missing mass $W^{*}$, backward proton momentum $\vec{p}_{s}$, and momentum transfer $Q^{2}$. The data are compared to a simple plane wave impulse approximation (PWIA) spectator model. A strong enhancement in the data observed at transverse kinematics is not reproduced by the PWIA model. This enhancement can likely be associated with the contribution of final state interactions (FSI) that were not incorporated into the model. Within the framework of the simple spectator model, a "bound neutron structure function" $F_{2 n}^{\text {eff }}$ was extracted as a function of $W^{*}$ and the scaling variable $x^{*}$ at extreme backward kinematics, where the effects of FSI appear to be smaller. For $p_{s}>0.4 \mathrm{GeV} / c$, where the neutron is far off-shell, the model overestimates the value of $F_{2 n}^{\text {eff }}$ in the region of $x^{*}$ between 0.25 and 0.6 . A dependence of the bound neutron structure function on the neutron's "off-shell-ness" is one possible effect that can cause the observed deviation.
\end{abstract}

DOI: 10.1103/PhysRevC.73.035212

PACS number(s): 24.85.+p, 25.30.-c, 21.45.+v

\section{INTRODUCTION}

Decades before the nucleon substructure was discovered, numerous models were developed that successfully describe most nuclear phenomena only in terms of nucleons, their excited states, and their strong force mediators-mesons. Nucleons and mesons are often called the "conventional" degrees of freedom of nuclear physics. The fundamental theory of strong interactions, quantum chromodynamics (QCD), describes physical processes in terms of quarks and gluons. QCD is very successful in describing the interaction of quarks at short distances, where perturbative methods, similar to those of quantum electrodynamics (QED) in atomic physics, are applicable. However, the same perturbative methods cannot

*Electronic address: klimenko@lanl.gov; current address: Los Alamos National Laboratory, Los Alamos, New Mexico 87545.

†Electronic address: skuhn@odu.edu

${ }^{\ddagger}$ Deceased.

${ }^{\S}$ Current address: University of New Hampshire, Durham, New Hampshire 03824.

"Current address: Old Dominion University, Norfolk, Virginia 23529.

"Current address: Massachusetts Institute of Technology, Cambridge, Massachusetts 02139.

${ }^{* *}$ Current address: Physikalisches Institut der Universitaet Giessen, D-35392 Giessen, Germany.

${ }^{\dagger \dagger}$ Current address: University of Massachusetts, Amherst, Massachusetts 01003. be applied to solve QCD at the length scales of a nucleus. The present difficulty in making rigorous predictions based on QCD at low momenta (corresponding to large distance scales) leaves us no choice but to continue to employ nuclear theories based on "effective" degrees of freedom-nucleons and mesons. In an attempt to resolve this discontinuity of theories, the focus of modern nuclear physics has turned to the intermediate region where QCD is not yet solvable, but the quark-gluon substructure of the nucleons must be taken into account in the nuclear models.

One example of the interface between a hadronic and a quark-based description is the (possible) modification of the (quark) structure of a nucleon that is part of a tightly bound pair. Because of the Heisenberg uncertainty principle, large momenta of the nucleons inside the nucleus can be associated with small internucleon spatial separations. The kinematic conditions are particularly clean in the case of the deuteron, where the relative motion of the two nucleons is completely described by the wave function in momentum space $\psi(p)$. In all models of the deuterium nucleus, the nucleons have mostly low momenta and therefore are relatively far apart. However, even in wave functions obtained from non-relativistic models of the nucleon-nucleon potential, there is the probability that nucleons have momenta so high that the proton and neutron can come very close together or even overlap. Such high-density configurations have been described by assuming that the quark distribution within a nucleon is modified either through off-shell effects [1] or through direct modification of the shape and size of the nucleon $[2,3]$. 
It has also been suggested that under these conditions, nucleons start to exchange quarks with each other or even merge into a single "six-quark bag" [4,5]. Quark-gluon degrees of freedom thus might play a direct role in modifying nucleon structure in high-density nuclear configurations. The analysis presented here is aimed at advancing the understanding of high-density, high-momentum nuclear matter.

To study these high-density configurations, we have used electron scattering from a high-momentum nucleon within a nucleus. In the case of a deuteron target, this can be easily verified by taking advantage of the inherently simple structure of the two-nucleon system. If all the momentum and energy is transferred to the neutron, the proton is a spectator to the reaction and recoils with its initial momentum. Assuming that the detected proton was indeed a spectator to the reaction, the initial momentum of the struck neutron can be obtained using momentum conservation. Thus the neutron is "tagged" by the backward-going spectator proton (for an extensive discussion of the spectator picture see, e.g., the papers by Simula [6] and Melnitchouk et al. [1]). Measurement of a high-momentum proton emitted backward relative to the momentum transfer direction allows us to infer that the electron interacted with a high-momentum neutron in deuterium.

\section{THEORETICAL MODELS}

\section{A. Nucleons in the nuclear medium}

If one could measure the total energy of the proton and that of the neutron bound within a deuteron separately at some instant $t$, then energy conservation would require that the sum of their energies equals the mass of the deuterium nucleus:

$$
E_{p}+E_{n}=M_{d} .
$$

At the same time, the mass of the deuteron is less than the mass of a free proton plus the mass of a free neutron, $M_{d}=$ $M_{p}+M_{n}-2.2246 \mathrm{MeV}$. Therefore, both the bound neutron and proton cannot be on-shell at the same time. In the "instant form" dynamics, one of the nucleons is assumed to be on-shell (the spectator), while the other one is off-shell, and its off-shell energy is $E^{*}=M_{d}-\sqrt{M_{s}^{2}+p_{s}^{2}}$.

The final state motion of the on-shell (spectator) nucleon can be described by its momentum $\vec{p}_{s}$ or the light-cone fraction $\alpha_{s}$,

$$
\alpha_{s}=\frac{E_{s}-p_{s_{\|}}}{M_{s}},
$$

where $p_{s}^{\mu}=\left(E_{s}, \vec{p}_{T}, p_{s_{\|}}\right)$is the spectator momentum 4vector. The component $p_{s_{\|}}$of the momentum is in the direction of the momentum transfer $\hat{q}$, and $\vec{p}_{T}$ is transverse to $\hat{q}$.

Using a nonrelativistic wave function $\psi_{\mathrm{NR}}\left(p_{s}\right)$, the "target density" of neutrons that are correlated with spectator protons of momentum $\vec{p}_{s}$ can be expressed as

$$
P\left(\vec{p}_{s}\right)=J\left|\psi_{\mathrm{NR}}\left(p_{s}\right)\right|^{2},
$$

where

$$
J=1+\frac{p_{s_{\|}}}{E_{n}^{*}}=\frac{\left(2-\alpha_{s}\right) M_{d}}{2\left(M_{d}-E_{s}\right)},
$$

is a flux factor that accounts for the motion of the struck nucleon.

The probability $P\left(\vec{p}_{s}\right)$ is related to the spectral function

$$
S\left(\alpha_{s}, p_{T}\right) \frac{d \alpha_{s}}{\alpha_{s}} d^{2} p_{T}=P\left(\vec{p}_{s}\right) d^{3} p_{s},
$$

which yields $S=E_{s} \cdot P\left(\vec{p}_{s}\right)$.

In the light-cone dynamics framework, a nonrelativistic deuterium wave function can be rescaled to account for relativistic effects at high momenta [2]:

$$
\begin{aligned}
S^{\mathrm{LC}}\left(\alpha_{s}, p_{T}\right) \frac{d \alpha_{s}}{\alpha_{s}} d^{2} p_{T} & =\left|\psi_{\mathrm{NR}}\left(|\vec{k}|^{2}\right)\right|^{2} d^{3} k, \\
\alpha_{s} & =1-\frac{k_{\|}}{\sqrt{M^{2}+\vec{k}^{2}}}, \\
\vec{p}_{T} & =\vec{k}_{T}, \\
|\vec{k}| & =\sqrt{\frac{M^{2}+p_{T}^{2}}{\alpha_{s}\left(2-\alpha_{s}\right)}-M^{2}},
\end{aligned}
$$

where $\alpha_{s}$ is the light-cone fraction of the nucleus carried by the spectator nucleon (with mass $M$ ) and $k^{\mu}=\left(k_{0}, \vec{k}_{T}, k_{||}\right)$is its internal momentum, with $k_{0}=\sqrt{M^{2}+\vec{k}^{2}}$. The relativistic effect, in this picture, manifests itself in that the measured momentum of the nucleon $p_{s_{\|}}$is rescaled in the laboratory frame from the internal momentum $k_{\|}$. The resulting deuterium momentum distribution is given by the spectral function

$$
S^{\mathrm{LC}}\left(\alpha_{s}, p_{T}\right)=\frac{\sqrt{M^{2}+\vec{k}^{2}}}{2-\alpha_{s}}\left|\psi_{\mathrm{NR}}(|\vec{k}|)\right|^{2} .
$$

The spectral function is normalized to satisfy the relation

$$
\iiint S^{\mathrm{LC}}\left(\alpha_{s}, p_{T}\right) \frac{d \alpha_{s}}{\alpha_{s}} d^{2} p_{T}=1 .
$$

In the plane wave impulse approximation (PWIA) spectator picture, the recoiling proton is on-shell at the moment of interaction and receives no energy or momentum transfer, so its initial and final momenta in the laboratory are the same. The differential cross section on a moving nucleon (with kinematics defined by the spectator variables $\alpha_{s}, p_{T}$ ) can then be calculated as

$$
\begin{aligned}
\frac{d \sigma}{d x^{*} d Q^{2}}= & \frac{4 \pi \alpha_{\mathrm{EM}}^{2}}{x^{*} Q^{4}}\left[\frac{y^{* 2}}{2(1+R)}+\left(1-y^{*}\right)\right. \\
& \left.+\frac{M^{* 2} x^{* 2} y^{* 2}}{Q^{2}} \frac{1-R}{1+R}\right] F_{2}\left(x^{*}, \alpha_{s}, p_{T}, Q^{2}\right) \\
& \times S\left(\alpha_{s}, p_{T}\right) \frac{d \alpha_{s}}{\alpha_{s}} d^{2} p_{T}
\end{aligned}
$$

where $S\left(\alpha_{s}, p_{T}\right) \frac{d \alpha_{s}}{\alpha_{s}} d^{2} p_{T}$ is the probability of finding a spectator with the given kinematics. In this expression, $F_{2}\left(x^{*}, \alpha_{s}, p_{T}, Q^{2}\right)$ is the off-shell structure function of the struck neutron and $R=\frac{\sigma_{L}}{\sigma_{T}}$ is the ratio between the longitudinal and transverse cross sections. The asterisk is used for variables that have been defined in a manifestly covariant way. For instance, the Bjorken scaling variable $x=\frac{Q^{2}}{2 M \nu}$ and the variable $y=\frac{v}{E}$ that are valid for the scattering from a free nucleon at 
rest are replaced with their counterparts for the scattering on a moving neutron inside the deuteron:

$$
\begin{aligned}
& x^{*}=\frac{Q^{2}}{2 p_{N}^{\mu} q^{\mu}} \approx \frac{Q^{2}}{2 M \nu\left(2-\alpha_{s}\right)}=\frac{x}{2-\alpha_{s}}, \\
& y^{*}=\frac{p_{N}^{\mu} q_{\mu}}{p_{N}^{\mu} k_{\mu}} \approx y,
\end{aligned}
$$

where $q^{\mu}=(v, \vec{q})$ is the momentum transfer 4-vector, $k^{\mu}=$ $(E, 0,0, E)$ is the momentum 4-vector of the incident electron, $p_{N}^{\mu}=\left(M_{d}-E_{s},-\vec{p}_{s}\right)$ is the momentum 4-vector of the offshell neutron, and $M_{d}$ is the mass of the deuterium nucleus. In this approximation, the struck nucleon is assumed to be on the energy shell, but off its mass shell. The mass of the free nucleon $\mathrm{M}$ is therefore replaced with the off-shell mass of the bound nucleon:

$$
M^{* 2}=\left(M_{d}-E_{s}\right)^{2}-\vec{p}_{s}^{2} .
$$

The invariant mass of the final hadronic state in ${ }^{2} \mathrm{H}\left(e, e^{\prime} p_{s}\right) X$ scattering can be expressed as

$$
\begin{aligned}
W^{* 2} & =\left(p_{n}^{\mu}+q^{\mu}\right)^{2}=M^{* 2}-Q^{2}+2\left(M_{d}-E_{s}\right) v+2 p_{s_{||}}|\vec{q}| \\
& =M^{* 2}-Q^{2}+2 M v\left(2-\frac{E_{s}-p_{s_{\|}}(|\vec{q}| / v)}{M}\right)
\end{aligned}
$$

where it was assumed that $M_{d} \approx 2 M$. In the (Bjorken) limit of $|\vec{q}| / \nu \rightarrow 1$, the fraction in the brackets of the last term in Eq. (13) takes the familiar form of the light-cone fraction of the nucleus carried by the spectator proton $\alpha_{s}=\frac{E_{s}-p_{s_{\|}}}{M}$, yielding

$$
W^{* 2} \approx M^{* 2}-Q^{2}+2 M v\left(2-\alpha_{s}\right) .
$$

If one assumes that $F_{2}$ is equal to its on-shell form, $F_{2}\left(x^{*}, \alpha_{s}, p_{T}, Q^{2}\right)=F_{2}^{\text {free }}\left(x^{*}, Q^{2}\right)$, and integrates over the spectator kinematics, one obtains the usual convolution result for the inclusive nuclear structure function $F_{2 A}$. In this picture, the nucleus is built from free nucleons; i.e., the struck nucleon has the same quark distribution as a free nucleon. Any observed modification of the cross section from that of a collection of free nucleons is just due to the kinematic rescaling [Eqs. (11)] because of the motion of the nucleons inside the nucleus. However, the difference in the $x$ dependence of the inclusive deep inelastic cross section for free and bound nucleons observed by the European Muon Collaboration (known as the EMC effect [7]) cannot be interpreted solely in terms of such a kinematic shift. A large number of models have been proposed to explain the EMC effect. A good review of this subject is given by Sargsian et al. [8].

A relatively "minimal" variation of the convolution result assumes that the bound nucleon structure is modified from the free one because the struck nucleon is off its mass shell $(E<M)$; for example, see Ref. [1]. Other models invoke a change of the nucleon size and therefore a rescaling of the structure function with momentum transfer $Q^{2}$, as in Ref. [3]. Frankfurt and Strikman [2] link the modifications to the structure function with a suppression of small (pointlike) valence configurations of a strongly bound nucleon. The most "radical" attempt to explain the EMC effect is that of Carlson and Lassila [4,5], where nucleons inside a nucleus in its high-density configuration are thought to merge and form multiquark states. For the case of deuterium, as much as $5 \%$ of the wave function would be in a 6-quark state in this model. The cross section for backward proton production is then expressed as a convolution of the distribution function for the valence quarks in a 6-quark cluster $V_{i}^{(6)}$ and the fragmentation function for the 5-quark residuum into a backward proton, $D_{p / 5 q}(z)$, with $z=\alpha /(2-x)$.

Although all of these models can describe at least some aspects of the EMC effect, they predict considerably different changes of the internal structure of deeply bound nucleons. These changes are masked in inclusive measurements, where one averages over all bound nucleons, most of which are below the Fermi surface. By selecting high-momentum nucleon pairs (with a fast backward-going spectator as "tag"), our experiment should be more sensitive to these possible modifications.

\section{B. Final state interactions}

The PWIA picture described above has to be modified to include the effect of final state interactions (FSI) and two-body currents (meson exchange currents). According to existing models (see below), there are kinematic regions where FSI are thought to be small, and other regions where FSI are enhanced. Reliable models of FSI exist for nucleon-nucleon rescattering [9]. In the resonant and deep inelastic region, the estimation of FSI is a lot more challenging. FSI can be modeled by replacing the spectral function in Eq. (10) with a distorted one: $S^{\mathrm{FSI}}\left(\alpha_{s}, \vec{p}_{T}\right)$.

Melnitchouk, Sargsian, and Strikman [1] use the $e D \rightarrow$ epn reaction as a first estimate of FSI in electron scattering from the deuteron. This calculation shows that for $\alpha_{s}>2-x$ and $\vec{p}_{T}$ close to zero, FSI are small. In this model, $S^{\mathrm{FSI}}$ is evaluated using a distorted wave impulse approximation (DWIA). According to this paper, FSI effects should not strongly depend on $x$, thus the ratios of the cross section for different ranges in $x$ should be a good tool to look for the EMC effect in the semiinclusive $e D \rightarrow e p X$ process. In the limit of large $x$, FSI become much more important for heavier nuclei, where rescattering hadrons produced in the elementary deep inelastic scattering (DIS) off the short-range correlation are dynamically enhanced. Therefore, deuterium targets, in the authors' opinion, provide the best way of studying the origin of the EMC effect.

A more recent publication by Ciofi degli Atti et al. [10] discusses backward proton production and FSI associated with DIS by evaluating $S^{\mathrm{FSI}}$ within a hadronization framework. The reinteraction of the backward-going spectator protons with the debris formed in a hadronization process is modeled using an effective cross section

$$
\sigma^{\mathrm{eff}}=\sigma^{N N}+\sigma^{\pi N}\left(n_{M}+n_{G}\right),
$$

where $\sigma^{N N}$ and $\sigma^{\pi N}$ are the total nucleon-nucleon and meson-nucleon cross sections, respectively, and $n_{M}$ and $n_{G}$ are the effective numbers of created mesons and radiated gluons. The cross section asymptotically tends to exhibit a simple logarithmic behavior. The magnitude of the effective reinteraction cross section differs significantly for different models, especially at angles of proton emission $\theta \sim 90^{\circ}$. This 
kinematic region is proposed by the authors as the best place to test various models of hadronization. In contrast with the calculation discussed in the beginning of the section, the model of [10] predicts significant FSI for proton momenta $\left|\vec{p}_{s}\right|>0.25 \mathrm{GeV} / c$ even at extreme backward angles.

\section{EXISTING DATA OVERVIEW}

Few data exist on the semiinclusive scattering of a lepton from deuterium with a recoiling nucleon in the backward direction with respect to the momentum transfer. The data published so far were taken using either neutrino or antineutrino beams and had very low statistics that do not allow detailed investigation of the cross sections of interest. These experiments (see Berge et al. [11] and Efremenko et al. [12]) focused on measuring the momentum, energy, and angular distributions of protons in the backward hemisphere relative to the beamline. Despite the low statistics, a notable difference in the distributions for backward and forward protons was observed. The data agreed well with a pair-correlation model in which the detected backward proton was assumed to be a spectator to the reaction.

The cross section ratio $\sigma^{\mathrm{Fe}} / \sigma^{D}$ measured by the European Muon Collaboration [7] (where $\sigma^{\mathrm{Fe}}$ and $\sigma^{D}$ are cross sections per nucleon for iron and deuterium, respectively) showed deviations from unity (now known as the EMC effect) that could not be explained only in terms of nucleon Fermi motion. That was the first evidence that the nuclear medium influences DIS processes. It provided an indication that nuclear matter is getting modified as its density increases. The effect was later confirmed by data from SLAC [13,14] and CERN [15].

An independent measurement of the modification of the quark structure of nuclei was made later at Fermilab [16] using continuum dimuon production in high-energy hadron collisions, known as the Drell-Yan process [17]. The measurement has shown no nuclear dependence in the production of the dimuon pairs in the region $0.1<x<0.3$ and, therefore, no modification of the antiquark sea in this range. A number of models developed to explain the EMC effect in terms of strong enhancement of the pion cloud were ruled out by this experiment.

Recent polarization transfer measurements by Dieterich and Strauch [18-21] in the ${ }^{4} \mathrm{He}\left(\vec{e}, e^{\prime} \vec{p}\right)^{3} \mathrm{H}$ reaction suggested medium modification of the electromagnetic form factors of the nucleon. The observed $10 \%$ deviation from unity could be explained by supplementing the conventional nuclear description with effects due to medium modification of the nucleon as calculated by the quark-meson coupling (QMC) model $[22,23]$. However, this conclusion is still under debate [24].

A model in which the neutron and proton form a single 6-quark cluster was recently tested [5] against old backward proton production data from neutrino scattering on deuterium collected at Fermilab [25]. These data had sufficient acceptance for backward protons but were not previously analyzed for this signal. The proton spectrum from neutrino and antineutrino scattering from deuterium, taken at CERN [26], was also discussed. The authors compared the momentum distribution of backward protons with the prediction of a 6-quark cluster model. Predictions of the model were in good agreement with the data; however, the statistics of the data were not sufficient to study the dependence on any other kinematic variables.

In summary, existing data on inelastic scattering off nuclei average over at least some of the relevant kinematic variables ( $x, Q^{2}$, and the momentum of the struck nucleon) and are often limited in statistics. Only a more detailed analysis of the dependence of the cross section on these variables can yield clear distinctions between different models and theoretical descriptions of nucleons bound in nuclei. The experiment on the reaction ${ }^{2} \mathrm{H}\left(e, e^{\prime} p_{s}\right)$ described here is the first to collect sufficient statistics for this purpose.

\section{EXPERIMENTAL SETUP}

The data were collected over a period of 46 calendar days in February and March of 2002 at the Thomas Jefferson National Accelerator Facility (TJNAF). We used a $5.75 \mathrm{GeV}$ electron beam with an average current of 6-9 nA. The experiment was staged in Hall B of the TJNAF, where the CEBAF large acceptance spectrometer (CLAS) is installed. Six superconducting magnetic coils divide CLAS into six sectors symmetrically located around the beamline. Each sector covers almost $60^{\circ}$ in azimuthal angle and between $10^{\circ}$ and $140^{\circ}$ in polar angle, thus providing almost $4 \pi$ acceptance for charged particles. CLAS sectors are equipped with identical sets of detector systems (Fig. 1): (1) three regions of drift chambers (DC) track the charged particle's passage though the region of the magnetic field, (2) a layer of scintillating paddles forms the CLAS time-of-flight (TOF) system, (3) the Cherenkov counters (CC) are installed in

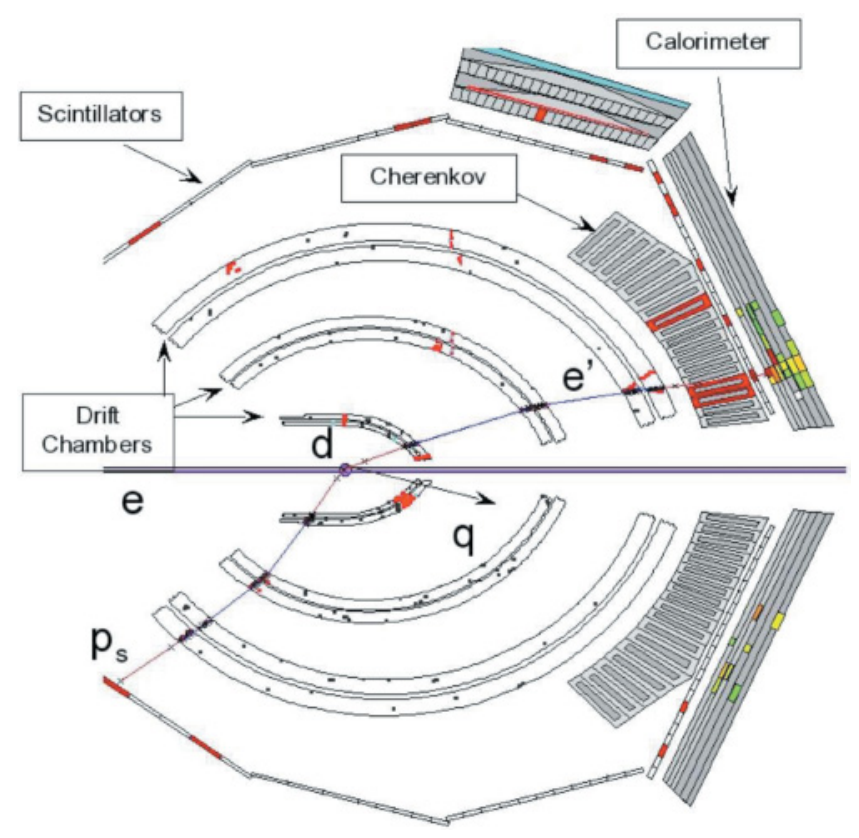

FIG. 1. (Color online) CLAS event with forward electron detected in coincidence with a backward proton. 


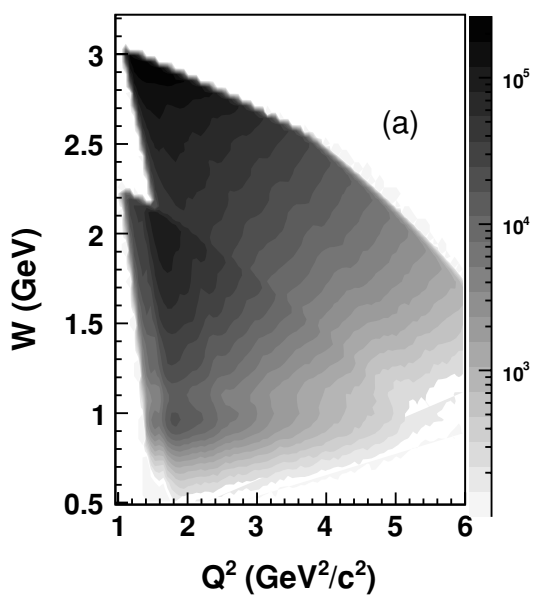

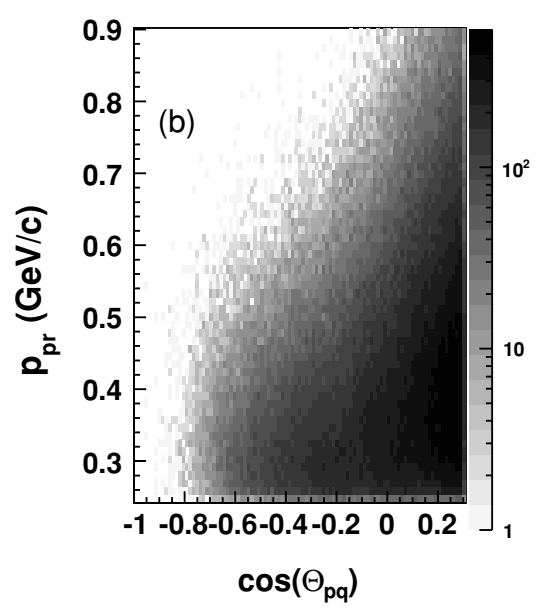

FIG. 2. Kinematic coverage for (a) electrons $\left(W\right.$ vs. $Q^{2}$ ) and (b) recoiling protons (momentum $p_{\text {pr }}$ vs. polar angle $\theta_{\mathrm{pq}}$ ), within fiducial cuts. the forward region $\left(10^{\circ}<\theta_{\text {lab }}<50^{\circ}\right)$ of the detector and efficiently discriminate electrons from pions up to the particle momenta $p \approx 2.7 \mathrm{GeV} / c$, and (4) several layers of lead and scintillating paddles form the electromagnetic calorimeter (EC) designed to separate electrons from minimum ionizing particles. CLAS is described in detail in Ref. [27].

A conical cryogenic $5 \mathrm{~cm}$ target, installed in the center of CLAS, was filled with liquid deuterium at a temperature of $22 \mathrm{~K}$ and pressure of $1315 \mathrm{mbar}$ with a density of $0.162 \mathrm{~g} / \mathrm{cm}^{3}$. The average beam current of $8 \mathrm{nA}$ produced a luminosity of $1.1 \times 10^{34} \mathrm{~cm}^{-2} \mathrm{~s}^{-1}$.

The CLAS trigger was formed by a coincidence between $\mathrm{CC}$ and EC. The signal level for the trigger coincidence was set to be at least 1 photoelectron in $\mathrm{CC}$ and $0.5 \mathrm{GeV}$ in $\mathrm{EC}$. The level 2 trigger required a DC track candidate in the sector of the calorimeter hit. With this trigger configuration, the data rate was about $3 \mathrm{kHz}$ and the dead time was usually less than $13 \%$.

Of the 4.5 billion events collected over the experimental run, only 350 thousand contained an electron in coincidence with a backward proton. The typical event of that type detected in CLAS is shown in Fig. 1. The collected data sample has wide coverage in kinematics of the electron and proton (Fig. 2). The momentum transfer $Q^{2}$ ranges between 1.2 and $5.5 \mathrm{GeV}^{2} / c^{2}$, while the invariant mass covers the quasielastic, resonant, and deep inelastic regions. Protons were detected at large angles relative to the momentum transfer vector $\vec{q}$, up to angles of $\theta_{\mathrm{pq}} \approx 145^{\circ}$ and with momenta above $0.28 \mathrm{GeV} / c$.

\section{DATA ANALYSIS}

In this section, we discuss all the key analysis steps that led to the extraction of the final results.

\section{A. Event selection}

The focus of this analysis is the $e d \rightarrow e^{\prime} p_{s} X$ reaction; therefore, events containing coincidences between the scattered electron and recoiling proton have to be selected first.

The scattered relativistic electron is expected to be the first particle that arrives at the detectors after interacting with the target nucleus. The particle was identified as an electron if it was the first in the event and its charge was measured by the DC to be negative. Electron identification (ID) cuts on the response of two of the remaining detector systems, CC and EC, reduce the background of $\pi^{-}$in the electron spectrum. The $\mathrm{CC}$ are very efficient in pion rejection up to $P \approx 2.7 \mathrm{GeV} / c$, where pions start to emit Cherenkov light. For lower momenta of the particle $P<3.0 \mathrm{GeV} / c$, a software cut of 2.5 photoelectrons was required to identify an electron. For the part of the data with particle momentum $P>3.0 \mathrm{GeV} / c$, a software cut of 1 photoelectron was used (and the fiducial region increased, see below) to increase acceptance. The electron produces an electromagnetic shower in the EC immediately after it enters, while pions mostly make a minimum ionizing signal with a small sampling fraction $(E / P)$. The minimum ionizing particles can be easily rejected by requiring that the visible energy deposited in the first 15 layers of the $\mathrm{EC}$ is $E C_{\text {inner }}>0.08 P$ and the total visible energy in the $\mathrm{EC}$ is $E C_{\text {total }}>0.22 P$.

In order to reduce the systematic uncertainty in the quality of electron identification, detector fiducial cuts are applied. The fiducial region of $\mathrm{CC}$ is known to be within the limits of the EC fiducial region; therefore, only a $\mathrm{CC}$ cut needs to be applied. We defined the fiducial region such that the $\mathrm{CC}$ was at least $90 \%$ efficient.

In addition to the particle charge information, the DCs also measure the length from the target to the TOF system and the curvature of the track. From the curvature of the track, the particle momentum can be reconstructed. The proton is identified using TOF time measurement $t_{\mathrm{TOF}}$, DC momentum $p_{\mathrm{DC}}$, and track length $r$ information. Assuming a positively charged particle is a proton, its velocity is given by

$$
v_{\mathrm{DC}}=\frac{p_{\mathrm{DC}}}{\sqrt{p_{\mathrm{DC}}^{2}+M_{p}^{2}}},
$$

where $M_{p}$ is proton mass. Then the time the proton travels from the target to the TOF system is $t_{\mathrm{DC}}=r / v_{\mathrm{DC}}$. The particle is identified as a proton if the time difference $\Delta t=t_{\mathrm{DC}}-t_{\mathrm{TOF}}$, corrected for the event start time, is within the time window of -3 to $7 \mathrm{~ns}$. At the lowest momenta (below $\approx 0.3 \mathrm{GeV} / c$ ), this time window is extended by a momentum-dependent amount of up to $3 \mathrm{~ns}$ to account for the extra transit time of protons losing a substantial fraction of their energy along their path. 
A vertex cut was applied to ensure that the interaction took place within the volume of the target. The electron was required to have a vertex $-2 \mathrm{~cm}<Z_{\mathrm{el}}<1.5 \mathrm{~cm}$, while the proton vertex cut was set to $-2.5<Z_{\mathrm{pr}}<2 \mathrm{~cm}$ (the target extends from -2.5 to $2.5 \mathrm{~cm}$ ). Additionally, the vertex difference between $Z_{\mathrm{el}}$ and $Z_{\mathrm{pr}}$ was required to be less than $1.4 \mathrm{~cm}$ to reduce the background from accidental coincidences.

\section{B. Kinematic corrections}

The geometric and structural complexity of CLAS is responsible for minor discrepancies in the measurement of the momentum and direction of a particle. These discrepancies are thought to be primarily due to the uncertainty in the magnetic field map and DC position. The effect of a displacement of the drift chambers and possible discrepancies in the measured magnetic field on the measured scattering angle $\theta_{\text {rec }}$ and momentum $p$ can be parametrized.

The correction function contains eight parameters describing the drift chamber displacements and rotations and eight parameters describing the possible uncertainties in the magnitude of the magnetic field on the path of the particle. These parameters can be determined using multiparticle exclusive reactions which are fully contained within the CLAS acceptance. In an exclusive reaction, all products of the reactions are detected and no mass is missing. Therefore, the kinematics of the reaction are fully defined and the goodness of fit can be evaluated using momentum and energy conservation. More details on this method can be found in Ref. [28].

For low-energy protons $(P<0.75 \mathrm{GeV} / c)$, energy loss in the target and detector is significant and needs to be corrected for. This energy loss was studied with the CLAS GEANT simulation, and an appropriate correction was applied to the data. The magnitude of the energy loss (and the appropriate correction) varies as a function of proton scattering angle and proton momentum in the range from $0.8 \%$ to $10 \%$ of the total proton momentum.

\section{Backgrounds}

Even after the ID cuts described above, pions remain a nonnegligible background in the electron spectrum. Their contribution needs to be estimated and appropriate corrections applied to the data. This was done using a sample of pions within EC cuts of $E_{\text {inner }}<0.05 \mathrm{GeV}$ and $E_{\text {total }}<0.1 \mathrm{GeV}$. The spectrum of photoelectrons in the Cherenkov counters of this pion sample was scaled such that the sum of the normalized spectrum and that of a "perfect" electron sample (from a simulation normalized to data within a tight EC cut) agreed with the measured Cherenkov spectrum for electron candidates within our regular EC cuts. This normalized pion spectrum was then integrated above the software ID cuts of 2.5 and 1.0 photoelectrons (depending on the data momentum range) and used to estimate the fraction of pions remaining in the electron sample after the Cherenkov ID cut. This fraction was fit to an exponential in pion energy, and the resulting estimate of the pion contamination (ranging to no more than $6 \%$ ) was used to correct the extracted data.

A similar technique was used to measure the rate of positrons relative to that of electrons, by taking positive charge tracks and fitting their energy spectrum in the EC with a combination of "pure" pions (based on Cherenkov response) and "golden electrons" (very high Cherenkov cut). This positron to electron ratio can be used to estimate the fraction of the detected electrons which were not scattered from the beam but came from pair production $\gamma \rightarrow e^{+} e^{-}$or the Dalitz decay $\pi^{0} \rightarrow \gamma e^{+} e^{-}$. Once again, an exponential fit to the ratio was used to estimate this contamination for all kinematic bins and to correct our final data accordingly.

Despite the vertex cuts, there is still a chance of having an accidental coincidence between an electron and a proton in the data sample. The background of accidentals has to be estimated and subtracted. At the same time, the loss of "true" protons due to the time and vertex cuts has to be determined. A purely accidental proton was defined as a positively charged particle with the time measured by the TOF system to be at least $12 \mathrm{~ns}$ longer than the expected TOF of a proton with that momentum. The time window for the accidental proton was taken to be the same as the proton ID time window, so that the expected arrival time for the accidental proton would not be more than 22 to at most $25 \mathrm{~ns}$ (at the lowest momenta) different from the expected arrival time of the real proton. For high momenta where the time window of accidentals is less than 5 ns away from when the deuteron (from elastic scattering events) would have arrived at the TOF counter, the accidental proton is defined to be within a $10 \mathrm{~ns}$ window starting at $5 \mathrm{~ns}$ after the expected arrival time of a deuterium ion. The average background of accidental coincidences per nanosecond of the proton time vertex was calculated from the rate in the "accidental time window" described above and compared with the unbiased data sample of coincidences with good proton particle ID (PID). The level of understanding of accidentals was also tested using the simulation results. The sum of the measured accidentals and the simulation is in agreement with the data on good electron-proton coincidences as selected by PID cuts (Fig. 3). A small discrepancy on the positive side of the $\Delta Z$ distribution is due to another type of unwanted coincidence where a particle originating from the first electron vertex reinteracts farther along the target cell, liberating a (backward) proton which arrives on time with respect to the TOF. Protons produced in such a way enhance the positive side of the vertex difference distribution. The selected

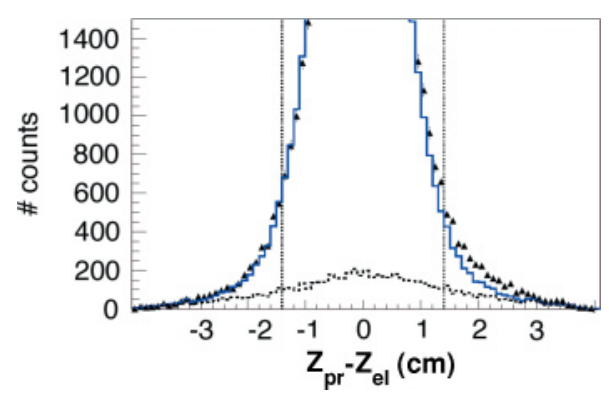

FIG. 3. (Color online) Data for the difference between the electron and proton vertex (triangles) compared to a fit (solid histogram) composed of a simulation of true coincidences (not shown) and measured accidental coincidences (dash dotted histogram). The vertical dashed lines indicate the cut used to select data for analysis. 
sample of accidentals contains only off-time events, and therefore does not fully reproduce the shape of the vertex difference distribution. A properly scaled sample of these excess events was added to the sample of purely accidental coincidences defined using off-time protons.

\section{Simulation}

To extract absolute results from our experimental data, the detector acceptance has to be evaluated and an appropriate correction applied to the data. An idealized model of all the detector systems of CLAS is implemented in the code known as GSIM. The program is built on the foundation of the GEANT simulation software package, supported by CERN. GSIM allows simulation of the detector response to a propagating particle, simulating energy loss as well as emission of secondary particles during the passage of the particle through parts of the detector. After the response of the ideal detector is simulated, existing detector inefficiencies are introduced. This is done using a separate program called GPP (GSIM post-processor). GPP uses precompiled information on dead regions of the DC and TOF system to remove the signal for these parts of CLAS from the GSIM output. The final output is then analyzed exactly the same way as the real data.

The events used as input for the CLAS GSIM simulation were generated following the cross section Eq. (10). The Paris wave function [29] was used to select the momentum of the "spectator" nucleon first. A comparison with the Argonne V18 wave function [30] showed a negligible difference in the momentum distributions. The generated nucleon momentum can either be directly used following the prescription for the nonrelativistic spectral function [Eqs. (3) and (4)] or as the "internal momentum" in the light-cone description, Eqs. (5)-(8). From the spectator nucleon kinematics, we then calculate the initial four-momentum of the struck nucleon, determine the scattered electron kinematics in the rest frame of that nucleon, and then transform it back to the laboratory frame. That way, all of the "starred" variables in Eq. (10) are automatically evaluated with the proper relativistic rescaling.

The electron scattering cross section used to generate the electron kinematics is based on the code RCSLACPOL developed at SLAC [31]. It uses parametrizations of world data on unpolarized structure functions and elastic form factors. These parametrizations are described in Ref. [32] and are based on fits to unpolarized structure function data from NMC [33] and SLAC [34-37]. The nucleon form factors were taken from Ref. [38]. All form factors and structure functions for bound nucleons are assumed to be equal to the free ones at the corresponding values of $x$ (in the DIS region) or $W$ (in the resonance region, with a smooth transition between both). The free neutron structure function $F_{2 n}$ was extracted from fits to the world data on the deuteron in a self-consistent manner by ensuring that our model, integrated over all spectator kinematics and summed over both proton and neutron contributions to electron scattering, agreed with those fits. Three different versions of the code were compiled to satisfy our needs for simulation of electron scattering on
${ }^{2} \mathrm{H}$ : (1) elastic scattering on one nucleon in the deuteron (with the other being a spectator), including the elastic radiative tail, (2) inelastic scattering on one nucleon in the deuteron (with and without radiative corrections), and (3) elastic scattering off the deuteron nucleus as a whole. Radiative effects can be included in the simulation following the prescription by Mo and Tsai [39]. In the first two cases, these radiative corrections are applied to the electron scattering cross section for the struck nucleon in its rest frame, while the spectator simply determines the kinematic transformation into the laboratory system. The generator is capable of simulating both inclusive ${ }^{2} \mathrm{H}\left(e, e^{\prime}\right)$ (by adding the first two processes for both protons and neutrons with the third one) and semiinclusive ${ }^{2} \mathrm{He}\left(e, e^{\prime} p_{s}\right)$ processes, which is controlled by a configuration file. While this generator may not be very realistic in its description of the underlying physical processes ( since it does not contain FSI, nonnucleonic currents in deuterium, or modifications of the nucleon structure function for off-shell nucleons), it is sufficiently accurate (see below) to allow a largely unbiased extraction of the acceptance and efficiency of CLAS, by comparing accepted simulated events to the initial distribution of generated events.

The quality of the simulation procedures can be evaluated by comparing the predicted number of counts for well-studied processes in data and simulation. To date, one of the best studied cross sections in nuclear physics is that of elastic electron scattering from a free proton. To select elastic events, a cut on the invariant mass $W$ was used: $0.9<W<1.1 \mathrm{GeV}$. The overall shape is reproduced well, and the measured cross section lies well within $10 \%$ of the simulated one at low $Q^{2}$ (where our statistical error allows a significant comparison). The $Q^{2}$ distribution of the simulated inclusive cross section for quasielastic scattering on deuterium is also in good agreement with the experimental data. Here, the events were also selected using the invariant mass cut $0.9<W<1.1 \mathrm{GeV}$. In the region of relatively good statistics at low $Q^{2}$, the deviation from unity on the data-to-simulation ratio does not exceed $10 \%$. Finally, the rate of inclusive ${ }^{2} \mathrm{H}\left(e, e^{\prime}\right) X$ events for all final state invariant masses $W$ agrees with the prediction of our model to within 5-10\%.

A sample of simulated events that exceeded the statistics of the experimental data by a factor of 10 was generated for the ${ }^{2} \mathrm{H}\left(e, e^{\prime} p_{s}\right)$ reaction and was used in the analysis to correct the data for detector acceptance and bin averaging effects. The high event count of the Monte Carlo ensures that the statistical error of the data points are not dominated by the statistical error of the simulation.

\section{E. Result extraction}

The events from the data set were sorted in fourdimensional kinematic bins in $W^{*}$ (or $x^{*}$ ), $Q^{2}, p_{s}$, and $\cos \theta_{\mathrm{pq}}$ (or $\alpha_{s}$ and $p_{T}$ ). We chose two bins in $Q^{2}$, one with $1.2 \leqslant Q^{2} \leqslant 2.1(\mathrm{GeV} / c)^{2}$ [average $Q^{2}=1.8(\mathrm{GeV} / c)^{2}$ ] and one with $2.1 \leqslant Q^{2} \leqslant 5.0(\mathrm{GeV} / c)^{2}$ [average $Q^{2}=$ $\left.2.8(\mathrm{GeV} / c)^{2}\right]$, and five bins in $p_{s}$, with average values of $p_{s}=0.3,0.34,0.39,0.46$, and $0.53 \mathrm{GeV} / c$.

To extract the final results, these bins were filled separately for the following categories of events: (1) experimental data with all the standard electron and proton ID cuts, (2) accidental 
electron-proton coincidences based on experimental data, (3) coincidences with protons from secondary scattering events, (4) simulated data for the elastic scattering on a bound neutron, including the radiative elastic tail, and (5) simulated data for the inelastic scattering on a bound neutron. Accidental coincidences and coincidences with secondary protons were then subtracted from the data on a bin-by-bin basis. The simulated elastic scattering data were also used to subtract the elastic radiative tail from the experimental data. For this purpose, both data and simulation were first integrated in the range of the invariant mass of the unobserved final state $W^{*}$ from 0.5 to $1.1 \mathrm{GeV}$. The elastic radiative tail in the simulation was then scaled by the ratio of the data to the simulation and subtracted.

As previously discussed, in the spectator picture, the cross section for the off-shell nucleon can be factorized as a product of the bound nucleon structure function and the nuclear spectral function, multiplied by a kinematic factor [see Eq. (10)]. Using the data of this experiment, it is possible to extract this product and, if a region can be identified where FSI are small and the spectral function is well described by the model, possibly extract the off-shell structure function by itself. To do that, the experimental data (with accidentals, rescattered proton events, and elastic radiative tail subtracted) were first divided by the simulated inelastic data. The simulated events were generated using the cross section Eq. (10) with full consideration of radiative effects. To extract the product of structure and spectral functions, the ratio of data to simulation was multiplied by the product $F_{2 n}\left(x^{*}, Q^{2}\right) \times S\left(\alpha_{s}, p_{T}\right)$, which was calculated using the same model used in the generator. Similarly, to obtain the product of the structure function $F_{2 n}$ with the probability distribution for the proton momentum in deuterium, we multiplied the ratio of data to simulation by the factor $F_{2 n}\left(x^{*}, Q^{2}\right) \times P\left(\vec{p}_{s}\right)$ from our generator model. In both cases, the dependence of the extracted data on the specific model for the simulation is minimized, since the "input" $\left[F_{2 n}\right.$ and $S\left(\alpha_{s}, p_{T}\right)$ or $\left.P\left(\vec{p}_{s}\right)\right]$ cancels to first order. Basically, this procedure corrects the data for the detector acceptance, bin migration, and radiative effects and produces a "normalized cross section" by dividing out the kinematic factor $\frac{4 \pi \alpha_{\mathrm{EM}}^{2}}{x^{*} Q^{2}}$ as well as the factor in square brackets in Eq. (10), which depends weakly on the ratio $R=\sigma_{L} / \sigma_{T}$. To extract the (off-shell) structure function $F_{2 n}^{\text {eff }}$, the ratio of data to simulation was multiplied by the free nucleon structure function $F_{2 n}\left(x^{*}, Q^{2}\right)$. This assumes that the spectral function used in the simulation describes the momentum distribution of the spectator protons reasonably well.

\section{F. Systematic uncertainties}

To simplify the statistical error calculation, all the corrections for the detector inefficiencies and data sample contamination (except for accidentals and the radiative elastic tail) were applied to the simulated events.

The efficiency of the $\mathrm{CC}$ electron ID cut is well reproduced in the simulation. A $1 \%$ systematic uncertainty enters here to account for the observed deviation of the cut efficiency from sector to sector. The EC ID cut efficiency is reproduced only partially. The efficiency of the cut in data was found to be $95 \%$; however, the same cut applied to the simulation is $98 \%$ efficient. The difference might be a result of data being contaminated with pions, despite the increased CC threshold. The simulated data were scaled down by a constant factor of 0.97 to account for the difference in the effect of the cut. A $2 \%$ systematic uncertainty was assigned to this factor because of the uncertainty about the source of the deviation. A variable factor that ranges from 1.06 to less than 1.01 was used to introduce pion contamination into the simulation. The factor varied with the particle scattering angle and momentum. A variable factor was also applied to the electron spectrum in the simulation to introduce electrons coming from electron-positron pair creation. The resulting systematic uncertainty was estimated by varying these factors by $50 \%$ of their deviation from unity. The resulting change in the distribution in each of the final histograms was used as an estimate of the systematic uncertainty of these corrections.

Some additional corrections were applied to the proton spectrum. A constant factor of 0.99 was introduced to reflect the difference in the effect of the proton timing ID cut on the real versus the simulated data. The systematic uncertainty of $0.5 \%$ on this number accounts for the momentum dependence of the effect. A factor dependent on the proton momentum was applied to the simulated data to account for the discrepancy between data and simulation in the effect of the cut that was set on the difference between the electron and proton vertices. The systematic uncertainty here is evaluated individually for each histogram, by varying the correction by $50 \%$. The accidental coincidences associated with secondary proton knock-out were corrected for on an event-by-event basis. We estimate the corresponding systematic error to vary from 0 to $6 \%$ with a mean of $2.3 \%$ (see Table I).

A major contribution to our systematic error comes from remaining differences between the simulated and the "true"

TABLE I. Systematic errors, in percent of data values, with typical range of the error and their rms values (in brackets).

\begin{tabular}{ll}
\hline \hline Source of uncertainty & $\begin{array}{c}\text { Typical range }(\% \text { of } \\
\text { data value })\end{array}$ \\
\hline EC ID cut & 2 \\
Trigger efficiency & 2 \\
Secondary electrons & 0.7 \\
Electron vertex ID cut & 0.6 \\
Proton timing ID cut & 0.5 \\
CC efficiency & 1 \\
Pion contamination & $0.5 \cdots 3$ \\
$e^{+} / e^{-}$contamination & $0 \cdots 0.75$ \\
Pure accidental coincidences & $0 \cdots\langle 1.2\rangle \cdots 4$ \\
Coincidences with knock-out proton & $0 \cdots\langle 2.3\rangle \cdots 6$ \\
Vertex difference cut & $0.75 \cdots 1.5$ \\
Quasielastic radiative corrections & $0 \cdots\langle 1.9\rangle \cdots 11$ \\
Inelastic radiative effects & $0 \cdots\langle 2.7\rangle \cdots 12$ \\
Luminosity & 3 \\
Tracking inefficiency & 11 \\
Bin migration \& model dependence of & $0 \cdots\langle 5.2\rangle \cdots 10$ \\
$\quad$ acceptance & \\
Total & $15.5 \cdots\langle 16.9\rangle \cdots 34.1$ \\
\hline
\end{tabular}


inefficiencies of CLAS. Even after removing bad channels and accounting for all known detector problems, we find that the ratio of simulated to measured rates for reconstructed protons varies from sector to sector. We use the root mean square (rms) variation between sectors to estimate this systematic error as about $11 \%$ on average. We also include a $3 \%$ scale error on the target density, effective target length, and beam charge calibration.

The data were corrected for the radiative elastic tail and accidental coincidences by direct subtraction of normalized (simulated or real) data (see previous subsection). The normalization factors were varied by $50 \%$ of their deviation from unity to estimate the systematic errors due to these corrections. The uncertainty on the inelastic radiative corrections was also calculated as $50 \%$ of the deviation from unity of the correction factor. We checked our radiative correction procedure against the existing code EXCLURAD [40] for the case of quasielastic scattering ( $p n$ final state) and found good agreement within the stated uncertainties.

A final systematic uncertainty comes from the model dependence of our simulated data. While the model input cancels in our extracted values for $F_{2 n}\left(x^{*}, Q^{2}\right) \times S\left(\alpha_{s}, p_{T}\right)$ to first order, both migration between adjacent kinematic bins and distribution of events within a bin (where the CLAS acceptance might vary) are somewhat model dependent. We estimated this effect by modifying the model input to agree with the cross section extracted from our data. The deviation of the simulated events with this modified cross section from the data is a direct measure of the magnitude of this systematic error. We found its magnitude to be generally below $5 \%$, going up to $10 \%$ for higher proton momenta.

All systematic errors were added in quadrature and are shown as shaded bands in the figures in the following section. The summary of systematic uncertainties is presented in Table I.

\section{RESULTS}

In the following, we show several representative histograms (one-dimensional projections of the four-dimensional bins), comparing our data to our simple PWIA spectator model to elucidate some general trends.

In Fig. 4, we show as a first step the accumulated number of protons (in coincidence with a scattered electron) for several bins in $\cos \theta_{\mathrm{pq}}$, where $\theta_{\mathrm{pq}}$ is the angle between the virtual exchanged photon and the proton. The data are not corrected for acceptance and efficiency and therefore fall off at large angles where CLAS has limited acceptance. The curves shown are from our simulation of these data, including the CLAS acceptance and without any normalization. Using the lightcone prescription [Eq. (8)] for the momentum distribution of the initial proton (solid curve), good agreement between the data and our Monte Carlo simulation is observed up to $\cos \theta_{\mathrm{pq}} \approx-0.3$. The result for the nonrelativistic wave function [Eq. (3), dashed line] is similar in these kinematics. At more forward angles, the data exceed the simulation by a large factor, especially at higher momenta [Fig. 4(b)], indicating a breakdown of the pure PWIA spectator picture. We assume
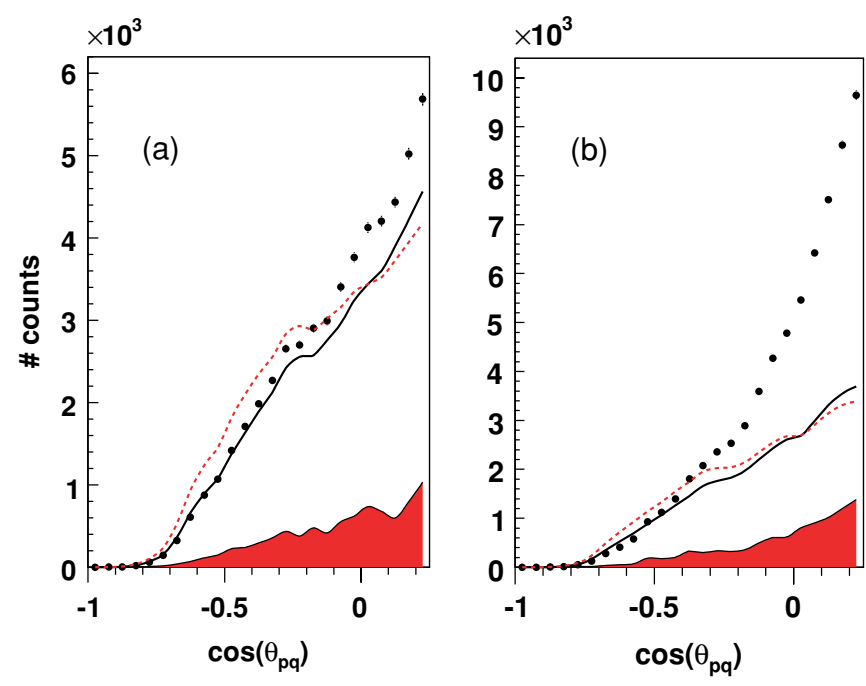

FIG. 4. (Color online) Data (points) and results of the Monte Carlo simulation based on two different PWIA models (solid and dashed curves) for the total number of counts vs $\cos \theta_{\mathrm{pq}}$ for proton momenta (a) $p_{s}=0.28-0.32 \mathrm{GeV} / c$ and (b) $p_{s}=0.36-0.42 \mathrm{GeV} / c$, integrated over electron kinematics. Total systematic error is indicated by the shaded band.

that this enhancement is due to FSI between the struck neutron and the spectator proton (see below).

The momentum distribution plotted separately for backward $\left(\theta_{\mathrm{pq}}>108^{\circ}\right)$ and transverse $\left(72^{\circ}<\theta_{\mathrm{pq}}<108^{\circ}\right)$ proton kinematics confirms this picture for the relative importance of non-PWIA processes (Fig. 5). The momentum distribution of the backward protons is reasonably well described by the PWIA model, indicating that distortions due to FSI are rather small in this region. At the same time, the yield for
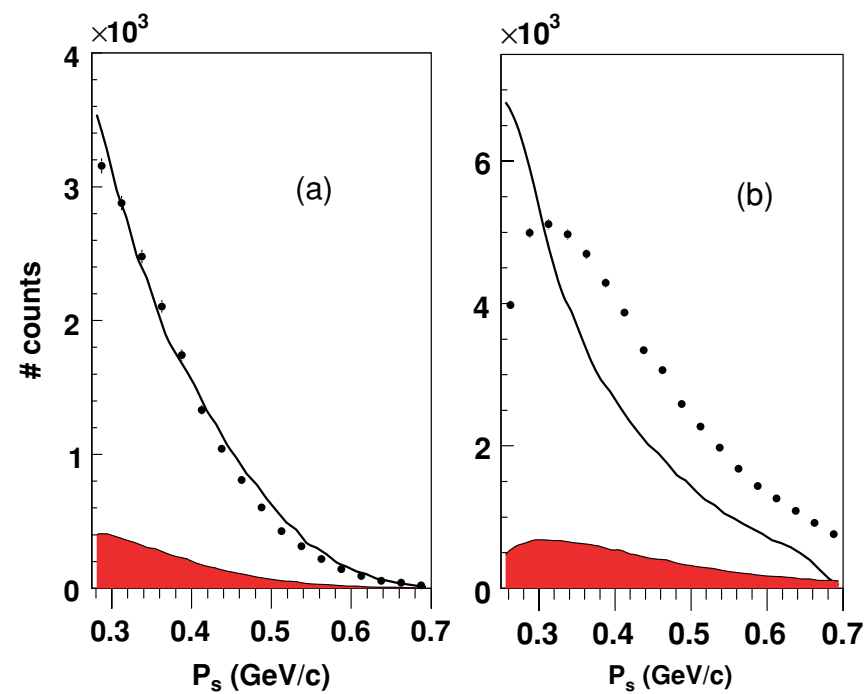

FIG. 5. (Color online) Momentum distribution of the recoiling proton. Data (points) are compared with our Monte Carlo simulation (solid curve) for two ranges of recoil angle: (a) $-1.0<\cos \theta_{\mathrm{pq}}<$ -0.3 and (b) $-0.3<\cos \theta_{\mathrm{pq}}<0.3$. Events were integrated over all missing masses and $Q^{2}$. 

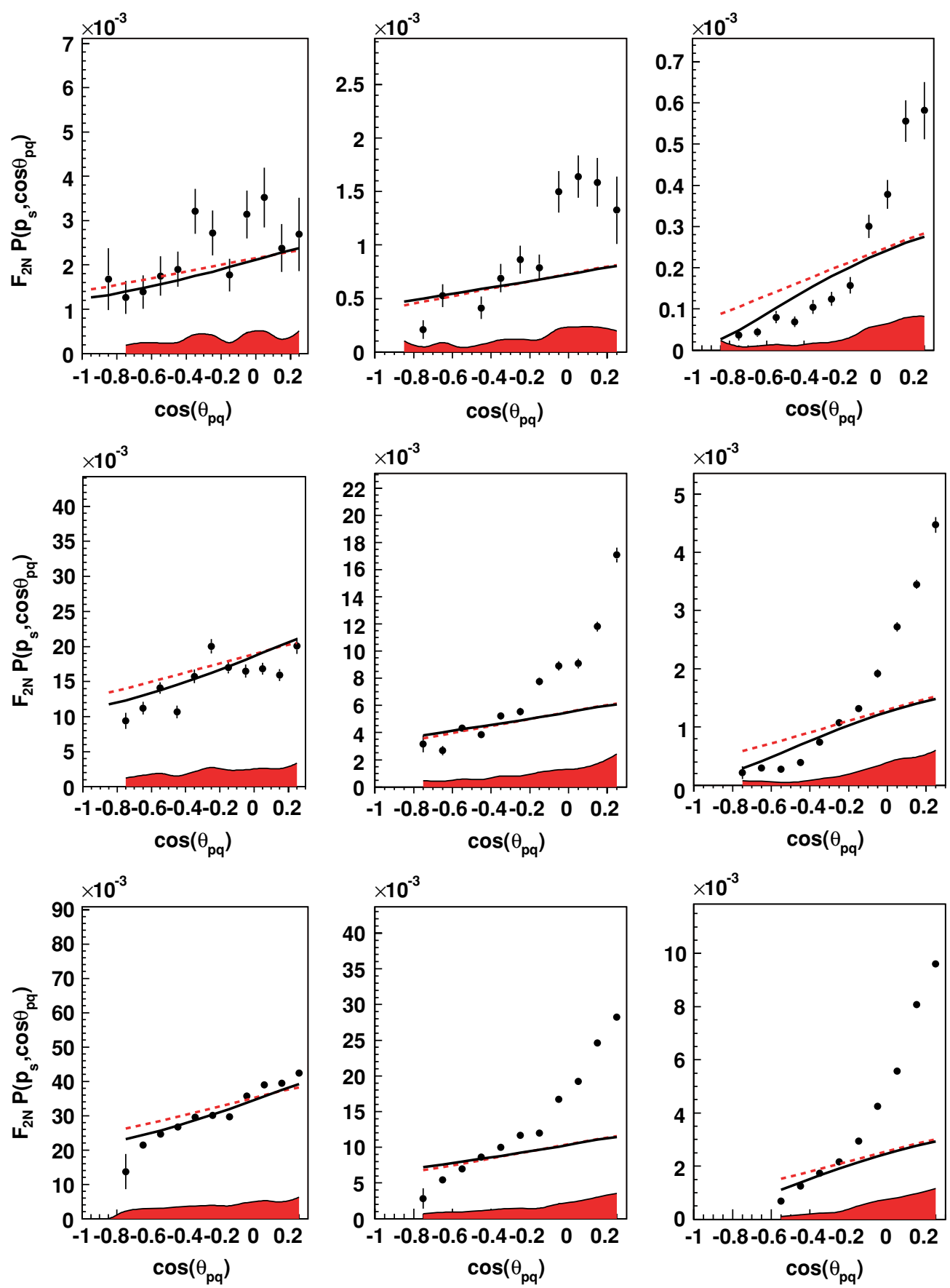

FIG. 6. (Color online) Results for the normalized cross section [equivalent to the product $F_{2 n} \times P\left(\vec{p}_{s}\right)$ in the spectator picture] for the reaction ${ }^{2} \mathrm{H}\left(e, e^{\prime} p_{s}\right) X$. Each row is for a different mass $W^{*}$ of the unobserved final state $X$, namely, $W^{*}=0.94 \mathrm{GeV}$ (quasielastic scattering) in the first row, $1.5 \mathrm{GeV}$ in the second, and $2 \mathrm{GeV}$ in the third. The three columns are for three different proton momentum ranges, with average momenta of $p_{s}=0.3,0.39$, and $0.56 \mathrm{GeV} / c$, from left to right, respectively. All data (filled circles with statistical error bars) are for our lower $Q^{2}$ bin [with average $Q^{2}$ of $1.8(\mathrm{GeV} / c)^{2}$ ]. The two lines come from our simple PWIA spectator model using a light-cone wave function (solid line) or a nonrelativistic one (dashed line); the shaded band at the bottom indicates the systematic error.

transverse protons is strongly enhanced at momenta above $0.3 \mathrm{GeV} / c$ while falling off at lower momenta, as predicted by several models of FSI $[1,9,10,41]$. At proton momenta below $0.28 \mathrm{GeV} / c$, the acceptance and efficiency of CLAS become more difficult to model exactly with our Monte Carlo simulation because of relatively large ionization losses. All data shown in this paper are therefore restricted to momenta above $0.28 \mathrm{GeV} / c$.

In Fig. 6, we look at the angular distribution of the protons in more detail. The reduced cross section described in the previous section is plotted for three different proton momenta (increasing from left to right) as well as three different missing 
mass ranges of the unobserved final state (increasing from top to bottom) in the reaction ${ }^{2} \mathrm{H}\left(e, e^{\prime} p_{s}\right) X$. Some trends can be observed:

(i) At proton momenta around $0.3 \mathrm{GeV} / c$, the extracted reduced cross section is consistent with our simple PWIA spectator model throughout the whole angular range and for all final state masses. This is consistent with expectations that destructive and constructive interference effects between FSI and PWIA cancel roughly in this momentum range $[9,41]$.

(ii) For larger proton momenta, deviations from PWIA behavior show up as an increase in the normalized cross section at transverse kinematics. This increase appears approximately around $\cos \theta_{\mathrm{pq}}=-0.3$ and continues beyond $\cos \theta_{\mathrm{pq}}=0\left(\theta_{\mathrm{pq}}=90^{\circ}\right)$. Such an increase is not likely due to uncertainties in the deuteron wave function, which is isotropic in the nonrelativistic case and is equal to the nonrelativistic wave function for transverse proton momenta if one uses light-cone wave functions. However, such an effect is expected within models of FSI because of the initial motion of the nucleon on which the rescattering occurs (see Fig. 3 in Ref. [9] and Ref. [41]). The strength of FSI in these models is the largest for the highest recoiling proton momenta, consistent with the trend of the data.

In Fig. 7, we study the dependence of FSI effects on the final state mass $W^{*}$ for one specific spectator momentum bin around $0.46 \mathrm{GeV} / c$. The figure shows the ratio between the observed cross section and the prediction of our PWIA spectator model for four different ranges in the final state missing mass (slightly offset from each other for each point in $\cos \theta_{\mathrm{pq}}$ ). The data for different missing mass values are statistically close to each other (and close to unity) in the backward region where rescattering effects can be assumed to be less important. Conversely, in transverse kinematics, the ratio substantially exceeds 1 and is largest for the highest $W^{*}$ bin. This behavior is in qualitative agreement with the FSI model by Ciofi degli Atti and collaborators [10,42], where the strength of rescattering is related to the size of the multihadron final system. The enhancement in transverse kinematics is also large in the $\Delta$-resonance region. This could be due to $\Delta$

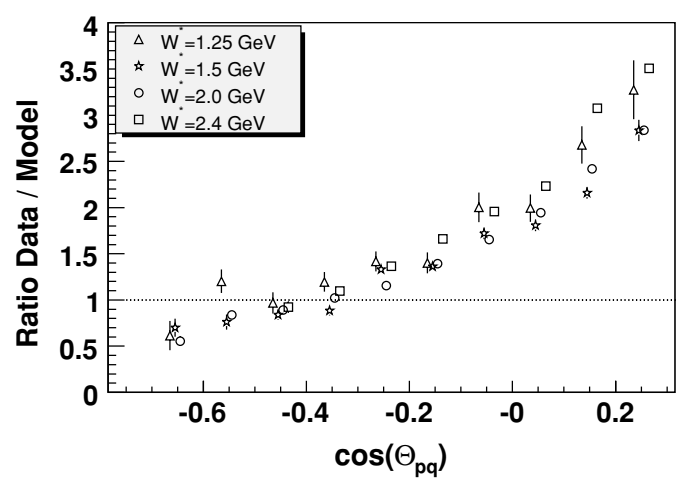

FIG. 7. Ratio of data to model as a function of $\cos \theta_{\mathrm{pq}}$ for four values of missing mass $W^{*}$ at $p_{s}=0.46 \mathrm{GeV} / c$ and $Q^{2}=$ $1.8 \mathrm{GeV}^{2} / c^{2}$. production in FSI between the struck neutron and the spectator proton.

We conclude that FSI effects seem to be most dominant in the region forward of $\cos \theta_{\mathrm{pq}} \approx-0.3$; while at larger angles, the angular distribution flattens out somewhat and the momentum distribution is closer to that expected from a simple PWIA model. In the following, we concentrate on this region to study the momentum (off-shell) dependence of the effective electron scattering cross section on the bound neutron. At first, we directly compare the extracted effective structure function of the off-shell neutron, $F_{2 n}^{\text {eff }}$, for inelastic final states $\left(W^{*}>\right.$ $1.1 \mathrm{GeV}$ ) to the on-shell structure function see Fig. 8), within our simple model [see Sec. V and Eq. (10)]. To obtain this structure function, the measured cross section, was divided by the proton momentum distribution according to the Paris potential wave function [29], the Mott cross section, and the kinematic factor as explained in the previous section. Even within the PWIA picture, the results could have a $p_{s}$-dependent scale error for each panel in Fig. 8, because our simple model may not perfectly describe the nucleon momentum distribution
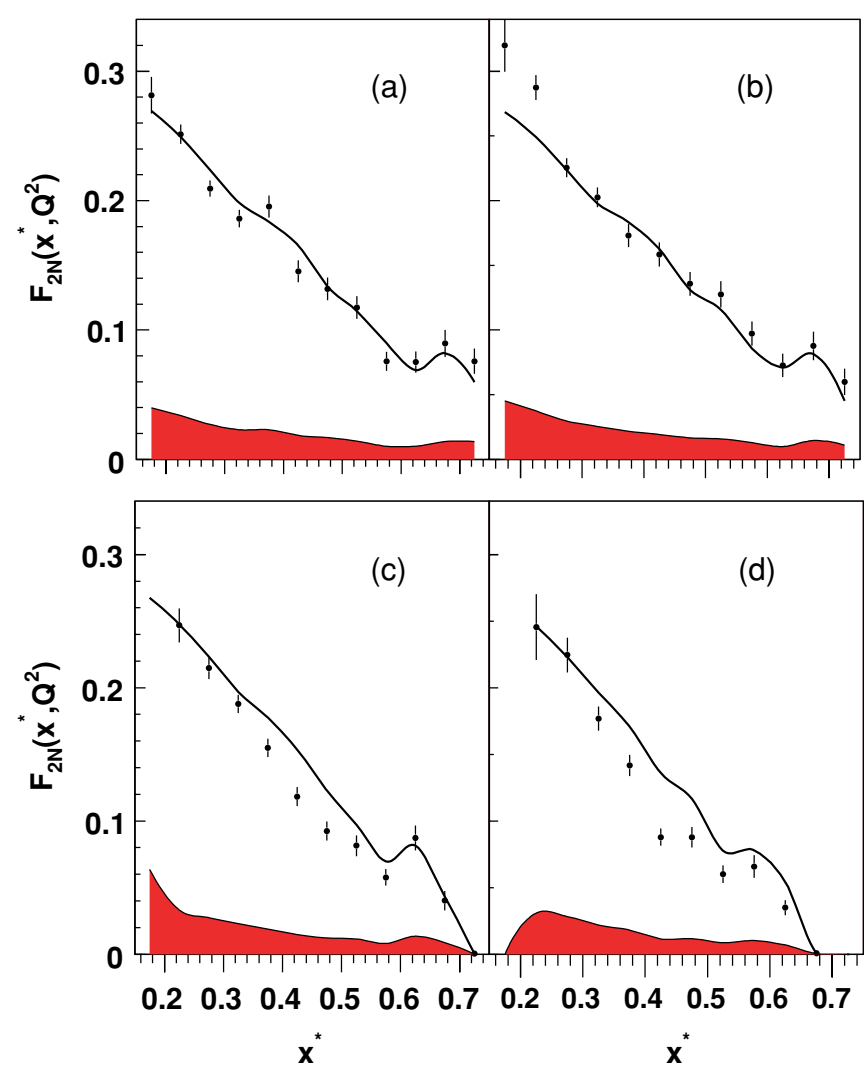

FIG. 8. (Color online) Results for the extracted off-shell structure function $F_{2 n}^{\text {eff }}$ of the neutron in the PWIA spectator picture. The model (solid curve) is a simple parametrization of the free on-shell neutron structure function, modified to account for the kinematic shift due to the motion of the off-shell neutron. The sections of the plot correspond to different recoiling proton momenta: (a) $p_{s}=0.3 \mathrm{GeV} / c$, (b) $p_{s}=$ 0.34 , (c) $p_{s}=0.46$, and (d) $p_{s}=0.56$. The quantity plotted here is similar (but not identical) to the quantity $F^{\text {(s.i.) }}$ defined in the paper by Simula [6]. The "bump" at large $x$ corresponds to the kinematics of $\Delta$-resonance excitation. 


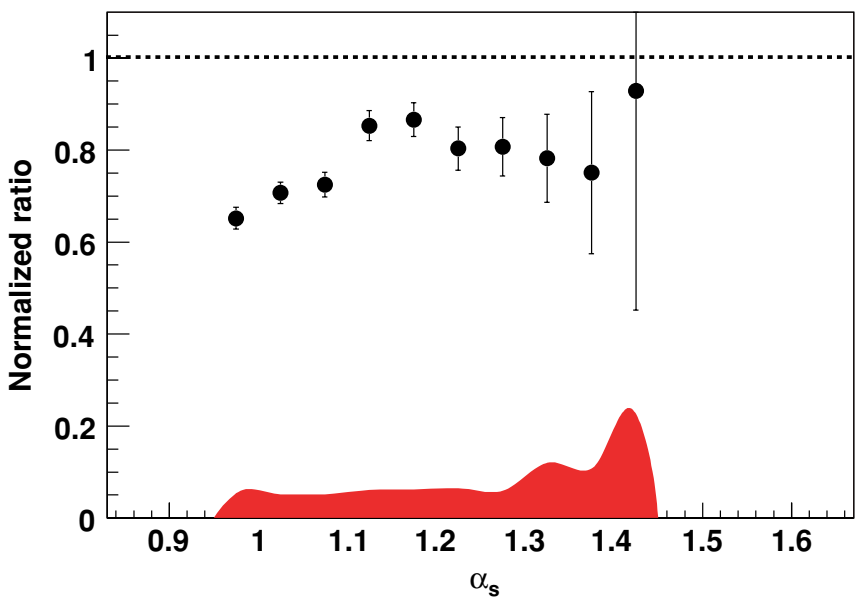

FIG. 9. (Color online) Ratio of the extracted off-shell structure function $F_{2 n}$ at $x=0.55, Q^{2}=2.8(\mathrm{GeV} / c)^{2}$ to that at $x=$ $0.25, Q^{2}=1.8(\mathrm{GeV} / c)^{2}$, divided by the ratio of the free structure functions at these kinematic points. Error bars are statistical only, shaded band indicates the overall systematic error. This plot is for kinematics similar (but not identical) to those in Fig. 6 in Ref. [1].

in deuterium; however, the $x^{*}$ dependence in each individual panel would be largely unaffected by such a scale error. Indeed, the data agree reasonably well with the simple parametrization of the free neutron structure function from our model at the two lower momenta (with average deviations of $\pm 10 \%$ ). At the higher two momenta, the data fall below the model in the range of $x$ between 0.3 and 0.6 by as much as $20-30 \%$. Some residual FSI might also contribute to the observed $x^{*}$ dependence, for instance, by enhancing the region of small $x^{*}$ (corresponding to large $W^{*}$ ).

To reduce the model dependence of such comparisons as in Fig. 8, the authors of Ref. [1] suggested taking the ratio between the extracted off-shell structure function at some relatively large value of $x^{*}$ (where most models predict the biggest off-shell effects) and that at a smaller value of $x^{*}$ where the EMC effect is known to be small. This ratio (normalized to the same ratio for the free neutron structure function $F_{2 n}$ ) is plotted in Fig. 9 for a range of transverse momenta, $0.25 \leqslant p_{T} \leqslant 0.35 \mathrm{GeV} / c$, versus the light-cone fraction $\alpha_{s}$. Within our PWIA model, the dependence on the proton momentum distribution $P\left(\vec{p}_{s}\right)$ cancels in this ratio since it enters the numerator and denominator at each point in the same way. There still remains an overall scale uncertainty due to the division by the ratio of $F_{2 n}$ for free neutrons at two different values of $x$, which is not perfectly well known. Furthermore, according to some models [10], FSI effects could be different for different $x^{*}$. This seems to be borne out by Fig. 9: While all PWIA models of off-shell effects predict unity for the ratio at values of the light-cone variable $\alpha_{s}$ around 1, we find a strong suppression in the region up to $\alpha_{s} \approx 1.1$ (corresponding to $\theta_{\mathrm{pq}}$ around $90^{\circ}$ ) where FSI effects are most pronounced. This behavior could be explained within the FSI model of Ref. [10] which predicts larger FSI effects for final states with a larger number of hadrons, leading to an increase in the denominator (cross section at small $x^{*}$, which corresponds to large energy transfer to the unobserved final state).
Beyond $\alpha_{s} \approx 1.1$, the data still lie below unity (by about $17 \%$ ) but appear to fall off only slowly with $\alpha_{s}$. Although this suppression could be interpreted as an off-shell effect, the data appear inconsistent with some of the more dramatic predictions of a steep falloff for the ratio at high $\alpha_{s}$ (e.g., Ref. [2]). The prediction for this ratio from the 6-quark cluster model [4] varies between 0.7 and 1 at $\alpha_{s}=1.4$ and is therefore compatible with our result. Once realistic calculations including FSI effects become available for the kinematics of our data set, a more quantitative comparison with various models for the off-shell behavior of the structure function $F_{2}\left(x^{*}, Q^{2}, p_{s}\right)$ will be feasible. Such calculations are underway $[41,43]$.

\section{SUMMARY}

Taking advantage of the large solid angle acceptance of the CEBAF large acceptance spectrometer, we collected a large amount of data $(\approx 350 \mathrm{~K}$ events $)$ on the reaction ${ }^{2} \mathrm{H}\left(e, e^{\prime} p_{s}\right) X$ in the exotic region of extreme backward proton kinematics. The data range from 1.2 to $5(\mathrm{GeV} / c)^{2}$ in momentum transfer $Q^{2}$ and reach values of the missing mass of the unobserved final state $W^{*}$ of up to $2.7 \mathrm{GeV}$. Protons with momentum $p_{s}$ as low as 0.28 and up to $0.7 \mathrm{GeV} / c$ were detected, at angles $\theta_{\mathrm{pq}}$ relative to the direction of the momentum transfer extending up to more than $140^{\circ}$. In terms of the light-cone variables, the data span values of the light-cone fraction $\alpha_{s}$ up to about 1.7, with a minimum proton transverse momentum relative to $\hat{q}$ of $0.15 \mathrm{GeV} / c$ and a maximum of $0.6 \mathrm{GeV} / c$.

Reduced cross sections were extracted as a function of $W^{*}$ (or Bjorken-variable $x^{*}$ ) and $\alpha_{T}, \vec{p}_{T}$ (or $\cos \theta_{\mathrm{pq}}, p_{s}$ ), for two large bins in $Q^{2}$, allowing us to test theoretical calculations against the presented data. Comparison with a simple PWIA spectator model shows moderately good agreement in the kinematic region of lower momenta and $\cos \theta_{\mathrm{pq}}<-0.3$. For increasing spectator momenta $p_{s}>0.3 \mathrm{GeV} / c$, FSI and other non-PWIA effects become strong, especially in the region of proton scattering angles $\cos \theta_{\mathrm{pq}}>-0.3$. These effects seem to depend on the invariant mass $W^{*}$; on the other hand, no strong dependence of these effects on momentum transfer $Q^{2}$ is observed. This behavior is in qualitative agreement with models $[10,42]$ that describe the strength of FSI in terms of the number of hadrons in the final state $X$. The angular $\left(\theta_{\mathrm{pq}}\right)$ and momentum $\left(p_{s}\right)$ dependence of the observed strength in the cross section in the quasielastic region (where $X$ is a neutron in its ground state) are also in good agreement with detailed calculations [41] showing a transition from destructive interference below $p_{s}=0.3 \mathrm{GeV} / c$ to a strong enhancement at $p_{s}>0.4 \mathrm{GeV} / c$ around $\cos \theta_{\mathrm{pq}}=0.2$ (see Fig. 6 and Ref. [44]).

A depletion compared to the PWIA model is observed in the data at $\cos \theta_{\mathrm{pq}}<-0.3$ and for high $p_{s}$, where the struck neutron is far off its mass shell. This reduction might be due to nucleon structure modifications. It is especially apparent in the region of moderate $x^{*}$ which overlaps in part with the nucleon resonance region. However, it is also possible that our simple model predicts too much strength in the deuteron momentum distribution at these higher momenta. This would lead to an 
"apparent" depletion for all values of $x^{*}$ (or $W^{*}$ ), which would be somewhat modified by a remaining FSI-induced enhancement at high $W^{*}$.

Ultimately, our data will serve to constrain detailed theoretical calculations, including off-shell and FSI effects. Once these effects are well understood at high spectator momenta, one can safely extract the neutron structure function at lower momenta where those corrections are smaller and where their uncertainty will not significantly affect the result. This method will be used in the "BoNuS" experiment at Jefferson Lab. A statistically improved data set with much larger kinematic coverage can be obtained once Jefferson Lab has been upgraded to a $12 \mathrm{GeV}$ beam energy.

\section{ACKNOWLEDGMENTS}

We would like to acknowledge the outstanding effort of the Accelerator, Target Group, and Physics Division staff at TJNAF that made this experiment possible. This work was supported by the U.S. Department of Energy, the Italian Istituto Nazionale di Fisica Nucleare, the U.S. National Science Foundation, the French Commissariat à l'Energie Atomique, the French Centre National de la Recherche Scientifique, and the Korea Science and Engineering Foundation. The Southeastern Universities Research Association operates the Thomas Jefferson National Accelerator Facility for the U.S. Department of Energy under DOE Contract DE-AC0584ER40150 Modification No. M175.
[1] W. Melnitchouk, M. Sargsian, and M. I. Strikman, Z. Phys. A 359, 99 (1997).

[2] L. L. Frankfurt and M. I. Strikman, Phys. Rep. 76, 215 (1981).

[3] F. E. Close, R. L. Jaffe, R. G. Roberts, and G. G. Ross, Phys. Rev. D 31, 1004 (1985).

[4] C. E. Carlson and K. E. Lassila, Phys. Rev. C 51, 364 (1995).

[5] C. E. Carlson, J. Hanlon, and K. E. Lassila, Phys. Rev. D 63, 117301 (2001).

[6] S. Simula, Phys. Lett. B387, 245 (1996).

[7] J. J. Aubert et al. (European Muon Collaboration), Phys. Lett. B123, 275 (1983).

[8] M. M. Sargsian et al., J. Phys. G 29, R1 (2003).

[9] L. L. Frankfurt, M. M. Sargsian, and M. I. Strikman, Phys. Rev. C 56, 1124 (1997).

[10] C. Ciofi degli Atti, L. P. Kaptari, and B. Z. Kopeliovich, Eur. Phys. J. A 19, 145 (2004).

[11] J. P. Berge et al., Phys. Rev. D 18, 1367 (1978).

[12] V. I. Efremenko et al., Phys. Rev. D 22, 2581 (1980).

[13] A. Bodek et al., Phys. Rev. Lett. 50, 1431 (1983).

[14] R. G. Arnold et al., Phys. Rev. Lett. 52, 727 (1984).

[15] G. Bari et al. (BCDMS Collaboration), Phys. Lett. B163, 282 (1985).

[16] D. M. Alde et al., Phys. Rev. Lett. 64, 2479 (1990).

[17] S. D. Drell and T.-M. Yan, Phys. Rev. Lett. 25, 316 (1970).

[18] S. Dieterich et al., Phys. Lett. B500, 47 (2001).

[19] S. Dieterich (Jefferson Lab E93-049 Collaboration and MAMI A1 Collaboration), Nucl. Phys. A690, 231 (2001).

[20] S. Strauch et al. (Jefferson Lab E93-049 Collaboration), Phys. Rev. Lett. 91, 052301 (2003).

[21] R. D. Ransome, Nucl. Phys. A699, 360 (2002).

[22] P. G. Blunden and G. A. Miller, Phys. Rev. C 54, 359 (1996).

[23] D. H. Lu, A. W. Thomas, K. Tsushima, A. G. Williams, and K. Saito, Phys. Lett. B417, 217 (1998).
[24] R. Schiavilla, O. Benhar, A. Kievsky, L. E. Marcucci, and M. Viviani, Phys. Rev. Lett. 94, 072303 (2005).

[25] T. Kafka et al. (E545 Collaboration), Bull. Am. Phys. Soc. 28, 756 (1983).

[26] E. Matsinos et al. (BEBC-WA59 Collaboration), Z. Phys. C 44, 79 (1989), and quotes therein.

[27] B. A. Mecking et al. (CLAS Collaboration), Nucl. Instrum. Methods A 503, 513 (2003).

[28] A. V. Klimenko and S. E. Kuhn, CLAS Note 03-005, Jefferson Lab, 2003 (unpublished).

[29] M. Lacombe, B. Loiseau, J. M. Richard, R. Vinh Mau, J. Cote, P. Pires, and R. de Tourreil, Phys. Rev. C 21, 861 (1980).

[30] R. B. Wiringa, V. G. J. Stoks, and R. Schiavilla, Phys. Rev. C 51, 38 (1995).

[31] K. Abe et al. (E143 Collaboration), Phys. Rev. D 58, 112003 (1998).

[32] P. L. Anthony et al. (E155), Phys. Lett. B493, 19 (2000).

[33] M. Arneodo et al. (NMC), Phys. Lett. B364, 107 (1995).

[34] A. Bodek et al., Phys. Rev. D 20, 1471 (1979).

[35] L. H. Tao et al. (E140X Collaboration), Z. Phys. C 70, 387 (1996).

[36] L. M. Stuart et al., Phys. Rev. D 58, 032003 (1998).

[37] K. Abe et al. (E143 Collaboration), Phys. Lett. B452, 194 (1999).

[38] P. E. Bosted, Phys. Rev. C 51, 409 (1995).

[39] L. W. Mo and Y.-S. Tsai, Rev. Mod. Phys. 41, 205 (1969).

[40] A. Afanasev, I. Akushevich, V. Burkert, and K. Joo, Phys. Rev. D 66, 074004 (2002); http://www.jlab.org/RC/exclurad/.

[41] J.-M. Laget, Phys. Lett. B609, 49 (2005); private communication.

[42] C. Ciofi degli Atti and B. Z. Kopeliovich, Eur. Phys. J. A 17, 133 (2003).

[43] C. Ciofi degli Atti, private communication.

[44] C. Butuceanu, Ph.D. thesis, College of William and Mary, 2005. 